\title{
DESCRIPCIÓN FONOLÓGICA DEL ROMANÉ DE CHILE
}

\author{
Alvaro González. \\ Gastón Salamanca
}

\begin{abstract}
RESUMEN
En este artículo se describen los fonemas segmentales del romané de Chile y su distribución. Además, se presenta un cuento gitano en transcripción fonémica.
\end{abstract}

\section{ABSTRACT}

This article describes the segmental phonemes and their distribution in Chilean Romané, the language of a gypsy ethnic minority. The phonemic transcription of a gypsy tale follows.

\section{Introducción}

Este trabajo presenta los fonemas segmentales del romané de Chile. Romané es el nombre que los gitanos rom de Chile dan a su lengua. Esta se opone al (ga)yikané (lengua de los no-gitanos (gayé)) y al ludar (lengua hablada por el grupo gitano boyás ${ }^{1}$ ).

\section{Breve introducción etnográfica al pueblo gitano ${ }^{2}$}

\subsection{Antecedentes generales}

\subsubsection{Origen y migraciones}

Según se ha demostrado, los gitanos tienen su origen en el sector noroccidental de la India. Desde allí emigraron alrededor del año mil d. C.

La aparición de los gitanos en Europa se estima alrededor del año 1400. Migraciones masivas a América se producen durante el siglo XIX.

\subsubsection{La lengua de los gitanos}

Cuando los gitanos emigraron de la India hablaban una misma lengua: el romaní. Hoy en día, sin embargo, tal homogeneidad lingüística se ha perdido. Dicho en palabras de Kenrick: 


\begin{abstract}
When the Romani (Gypsy) people left India....they took with them their language, and this language is still used by over five million persons, ranging from the settled Gypsies of Eastern Europe to the nomadic

Kalderash of Western Europe and the Americas. In a few instances the spoken Romani language itself has died out, as with the Boyash in Romania, in others, it survives only as lexis, using the syntax, morphology of the host language... (Kenrick 1979: 110. En van der Voort 1996: 2).
\end{abstract}

\title{
1.2. Gitanos en Chile ${ }^{3}$
}

\subsubsection{Procedencia y población actual}

Las primeras familias gitanas que llegaron a Chile, procedían en su mayoría de Serbia (Dunin 1989). A la inmigración serba, de acuerdo a Sotomayor (1993), hay que agregar un pequeño número de inmigrantes rumanos, rusos y norafricanos.

El número de gitanos que reside actualmente en Chile se estima entre 5000 y 8000 .

\subsubsection{Grupos}

Existen dos grandes grupos:

Grupo romá (habla romané)

Grupo ludar o boyas (habla algún dialecto rumano)

Es interesante consignar que la interacción entre los gitanos romá y ludar se produce sólo en español, pues los romá no hablan ludar y viceversa.

\section{Marco teórico y formato de presentación}

Tanto el marco teórico para el análisis de los datos como el formato de presentación de este artículo los hemos extraído de Pike 1947.

\section{Fonemas segmentales y alófonos del romané de Chile}

Los fonemas del /rromané/ de Chile son: /i, e, a, o, u, p, b, ph, t, d, th, k, g, kh, f, v, s, $\mathrm{z}, \mathrm{x}, \check{c}, \mathrm{dz}, \mathrm{c} h, \mathrm{~m}, \mathrm{n}, \tilde{\mathrm{n}}, \mathrm{l}, \mathrm{r}, \mathrm{rr}, \mathrm{j}, \mathrm{w} /$.

\subsection{Vocales}

Fonema

/i/
Alófonos

[i]

alta, cerrada, anterior, no redondeada.
Ejemplos

/inčá/ 'allá' [in,.čá]

/číro/ 'tuyo' [čí.ro]

/kávi/ 'olla' [ká.vi] [ká.bi] 


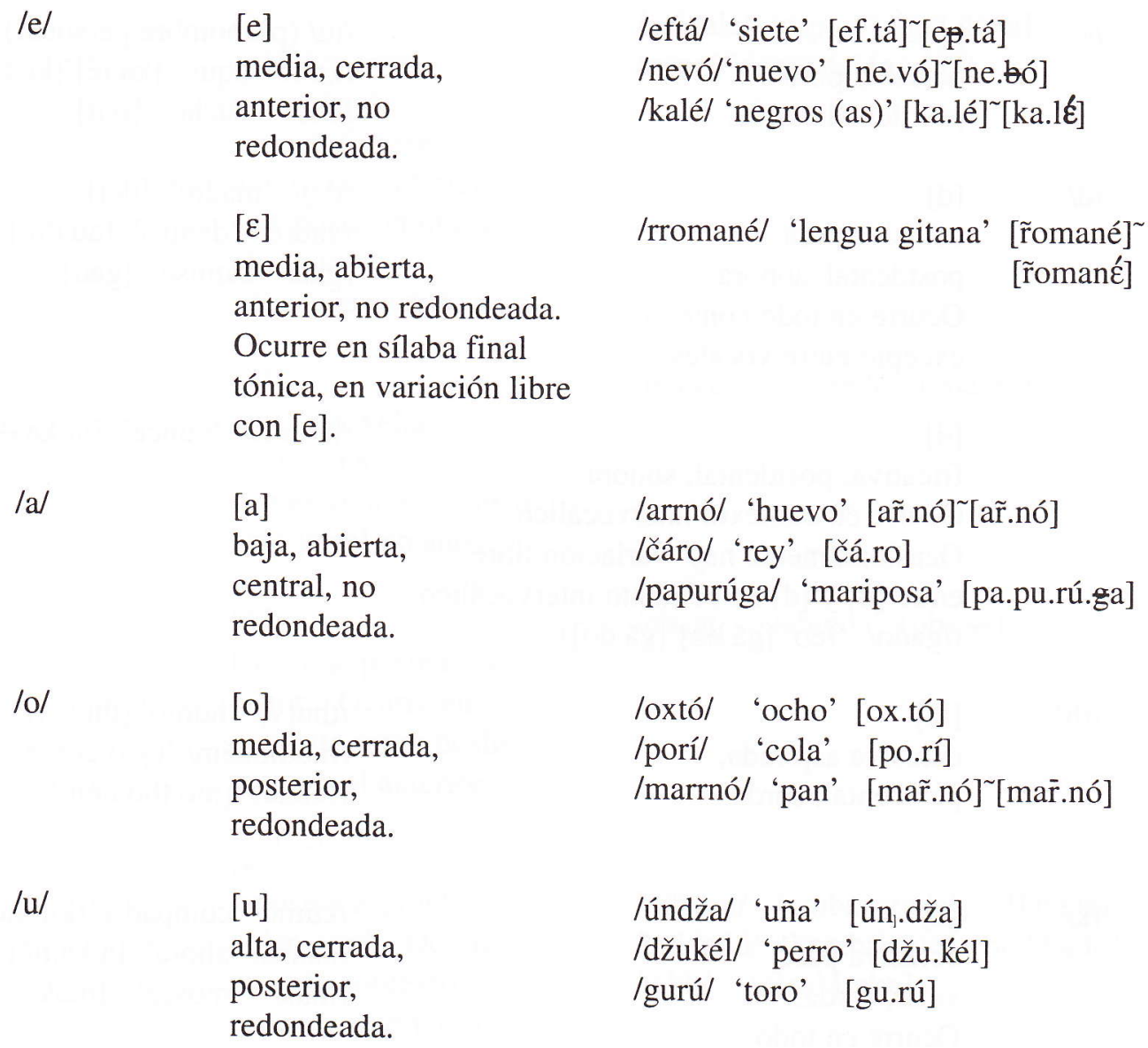

[E]

media, abierta, anterior, no redondeada.

Ocurre en sílaba final tónica, en variación libre con [e].

/a/

[a]

baja, abierta, central, no redondeada.

leftá/ 'siete' [ef.tá] [ep.tá] /nevó/‘nuevo’ [ne.vó] [ne.bó] /kalé/ 'negros (as)' [ka.lé] [ka.lé]

/rromané/ 'lengua gitana' [r̃omané] [romané]

$10 /$

[o]

media, cerrada,

posterior, redondeada.

/arrnó/ 'huevo' [ar̃.nó] [ar̃.nó]

/čáro/ 'rey' [čá.ro]

/papurúga/ 'mariposa' [pa.pu.rú.ga]

/u/

[u]

alta, cerrada, posterior, redondeada.

/oxtó/ 'ocho' [ox.tó]

/poríl 'cola' [po.rí]

/marrnó/ 'pan' [mar̃.nó] [mar̄.nó]

/úndža/ 'uña' [ún.ddža]

/džukél/ 'perro' [džu.kél]

/gurú/ 'toro' [gu.rú]

\subsection{Consonantes}

\section{Fonema \\ $/ \mathrm{p} /$ \\ Alófono
[p]
oclusiva pura,
bilabial, sorda.}

$/ \mathrm{b} /$

ph/ [b]

oclusiva pura,

bilabial, sonora.

[ph]

oclusiva aspirada,

bilabial, sorda.

\section{Ejemplos}

/pápo/ 'abuelo' [pá.po]

/kapúto/ 'casaca' [ka.pú.to]

/sap/ 'serpiente' [sap]

/baró/ 'grande' (masc.) [ba.ró]

/drabá/ 'remedios' [dra.bá]

/čib/ 'lengua' [čib]

/phej/ 'hermana' [phei]

$* / V_{-}$VI

*I_\#I 
(t) $\quad[\mathrm{t}]$

oclusiva pura

postdental, sorda.

/d/ [d]

oclusiva pura

postdental, sonora.

Ocurre en todo contexto,

excepto entre vocales.

[A]

fricativa, postdental, sonora.

Ocurre en contexto intervocálico.

Ocasionalmente hay variación libre

entre [e] y [d] en contexto intervocálico

(/gado/ 'feo' [gá.ło] [gá.do]).

/th/ [th]

oclusiva aspirada,

postdental, sorda.

/k/ [k]

oclusiva pura,

velar, sorda.

Ocurre en todo

contexto, excepto

ante vocal anterior.

[k]

oclusiva pura,

postpalatal,

sorda. Ocurre

ante vocal anterior.

/g/ [g]

oclusiva pura,

velar, sonora.

Ocurre ante vocal

no anterior:

- en posición inicial

absoluta;

- tras consonante nasal. /tu/ (pronombre personal) [tu]

/katél 'aquí' [ka.té] [ka.té]

/but/ 'mucho' [but]

/dej/ 'madre' [dei]

landrél 'dentro' [an.dré]

/gad/ 'camisa' [gad]

/níkada/ 'nunca' [ní.ka.ła]

/thu(v)/ 'humo' [thu(v)]

/mothodém/ '(yo) conté

(relaté)' [mo.tho.dém]

*I_\#!

/kúmo/ 'compadre'[kú.mo]

lakanál 'ahora' [a.ka.ná]

/mak/ 'mosca' [mak]

$/$ kisí/ ${ }^{4}$ [ki.sí]

/sukí/ 'flaca' [su.kí]

/gálveno/ 'amarillo’ [gál.ve.no]

/čingó/ 'mojado' [čin.gó]

/čang/ 'diente' [čang] 
[ǵ]

oclusiva pura,

postpalatal, sonora. Ocurre

ante vocal anterior:

- en posición inicial

absoluta;

- tras consonante nasal.

[q]

fricativa, velar

sonora. Ocurre en

contexto intervocálico

ante vocal no anterior.

[8́⿴囗十)

fricativa, postpalatal, sonora. Ocurre en contexto intervocálico

ante vocal anterior.

/kh/

/f/

[kh]

oclusiva aspirada, velar, sorda. Ocurre en todo contexto, excepto ante vocal anterior.

[k'h] oclusiva aspirada, postpalatal, sorda. Ocurre ante vocal anterior.

[f]

fricativa, labiodental, sorda.

[p]

fricativa, bilabial sorda. Variación libre entre $[\mathrm{f}]$ y $[\mathrm{p}]$. /geldála/ 'espejo’ [ǵel.dá.la]

/nangíl'desnuda' [naý.ǵí]

/phagó/ 'quebrado' [pha.gó]

/phagí/ 'quebrada' [pha.ǵ́i

[/khamí/ 'embarazada'] [kha.mí] /bokhaló/ 'hambriento' [bo.kha.ló] /jakh/ 'ojos' [jakh]

/dikhé/ ‘(tú) ves’ [di.ḱhé] /fála/ 'gracias' [fá.la] [pá.la]

/kafával 'café' [ka.fá.va] [ka.pá.ba]

*/_\#/ 


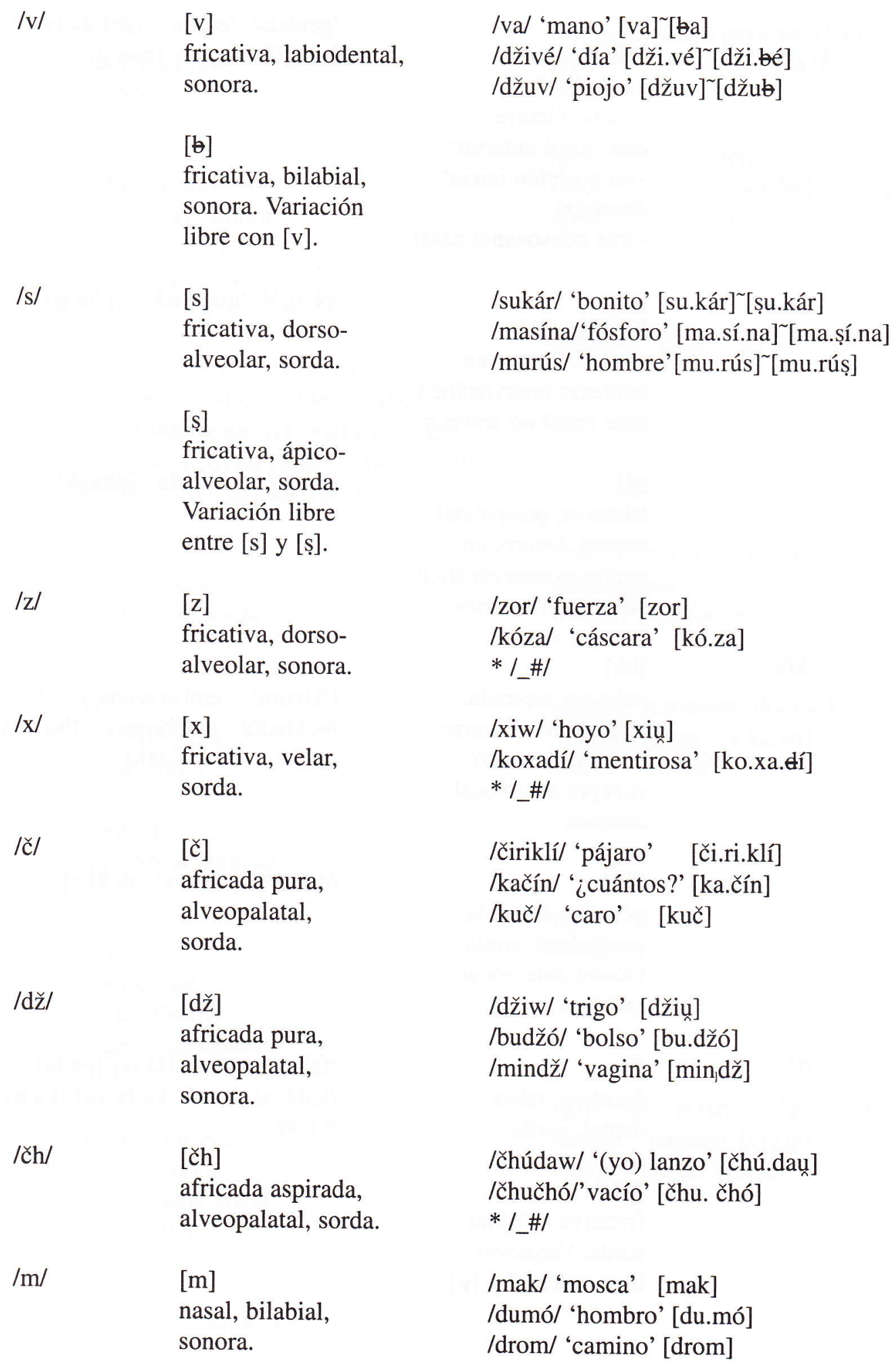

/čh/

[čh] africada aspirada, alveopalatal, sorda.

$/ \mathrm{m} /$

[m] nasal, bilabial, sonora.

$/ \mathrm{va} /$ 'mano' [va] [ba]

/dživé/ 'día' [dži.vé] [dži.bé]

/džuv/ 'piojo' [džuv]'[džub]

/sukár/ 'bonito' [su.kár] [șu.kár] /masína/'fósforo' [ma.sí.na] [ma.șína] /murús/ 'hombre'[mu.rús] [mu.rúş]

/Čiriklí/ 'pájaro' [či.ri.klí] /kačín/ ‘ ¿cuántos?' [ka.čín] /kučl 'caro' [kuč]

/džiw/ 'trigo' [džiun]

/budžó/ 'bolso' [bu.džó]

/mindžl 'vagina' [min,dž]

/čhúdaw/ '(yo) lanzo' [čhú.daun]

/čhučhó/'vacío' [čhu. čhó]

*I_\#I

/mak/ 'mosca' [mak]

/dumó/ 'hombro' [du.mó]

/drom/ 'camino' [drom] 
$/ \mathrm{n} /$

[n]

nasal, alveolar,

sonora. Ocurre

ante vocal y ante

consonante alveolar.

[n]

nasal, postdental, sonora. Ocurre ante consonante postdental.

$\left[\mathrm{n}_{1}\right]$ nasal, alveopalatalizada, sonora. Ocurre ante consonante alveopalatal.

[ń]

nasal, postpalatal, sonora. Ocurre ante consonante postpalatal.

[y]

nasal, velar, sonora.

Ocurre ante consonante velar.

$/ \tilde{\mathrm{n}} /$

/1/

$/ \mathrm{r} /$

/rr/ [ñ]

nasal, dorsopalatal, sonora.

[1] lateral, alveolar, sonora.

[r] vibrante simple, alveolar, sonora.

[ī] vibrante múltiple, alveolar, sonora. /nak/ 'nariz' [nak] /sunó/ 'sueño' [su.nó] /kolín/ 'pecho' [ko.lín]

/dandá/ 'dientes' [daṇ.dá]

/ivénd/ 'invierno' [i.vénd] [i.bénd]

/khandžirí/ 'iglesia' [khan,dži.rí] /panč/ 'cinco' [pan,č]

/nangí/ 'desnuda' [naú.gúí]

/bangó/ 'cojo' [ban.gó]

/sing/ 'cuerno' [sing]

/ñaño/ (nombre propio) [ña.ño]

/puñava/'alfombra'

[pu.ñá.va] [pu.ña.ła]

*I\#I

/lové/ 'dinero' [lo.vé] [lo.bé]

/soló/ 'cordel' [so.ló]

/sil/ 'frío' [sil]

/rovlí/ 'bastón' [ro.vlí] [ro.blí]

/móra/ 'mar' [mó.ra]

/sir/ 'ajo' [sir]

/rromí/ 'esposa' [r̃o.mí] [r̃o.mí]

/korró/ 'ciego' [ko.r.ró] [ko.,̃ó]

/barr/ 'piedra' [bar̃ $]^{\sim}[\mathrm{bar}]$ 


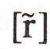

vibrante múltiple,

uvular, sonora.

\subsection{Semiconsonantes}

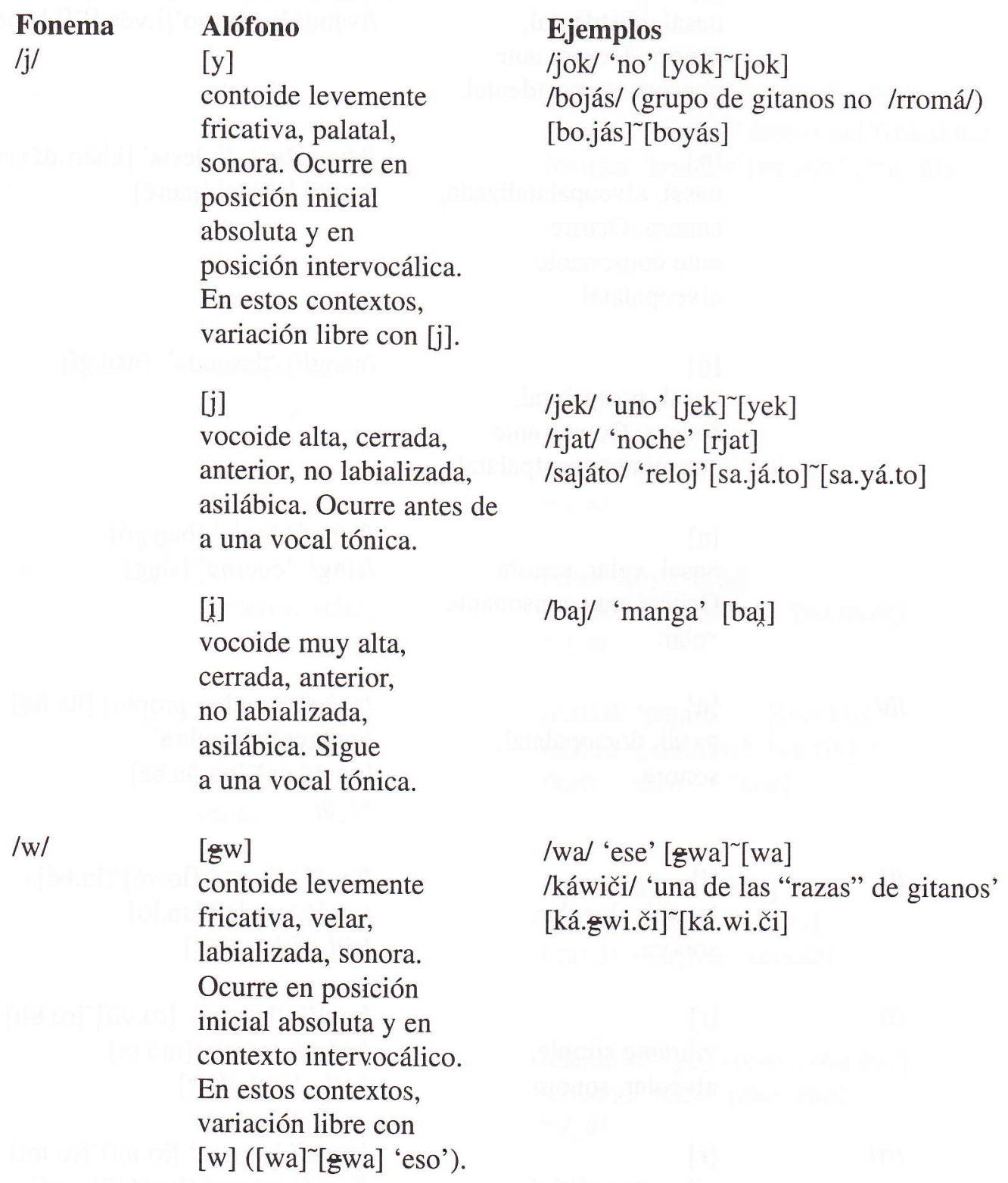

Fonema

/j/

/w/

Alófono

[y]

contoide levemente

fricativa, palatal,

sonora. Ocurre en

posición inicial

absoluta y en

posición intervocálica.

En estos contextos,

variación libre con [j].

[j]

vocoide alta, cerrada, anterior, no labializada, asilábica. Ocurre antes de a una vocal tónica.

[i]

vocoide muy alta, cerrada, anterior, no labializada, asilábica. Sigue

a una vocal tónica.

[gw]

contoide levemente

fricativa, velar, labializada, sonora. Ocurre en posición inicial absoluta y en contexto intervocálico.

En estos contextos, variación libre con [w] ([wa] [zwa] 'eso').

\section{Ejemplos}

/jok/ 'no' [yok] [jok]

/bojás/ (grupo de gitanos no /rromá/)

[bo.jás] [boyás]

/jek/ 'uno' [jek] [yek]

/rjat/ 'noche' [rjat]

/sajáto/ 'reloj'[sa.já.to] [sa.yá.to]

/baj/ 'manga' [bai]

/wa/ 'ese' [gwa] [wa]

/káwiči/ "una de las "razas" de gitanos'

[ká.gwi.či] [ká.wi.či] 
[w] /naswaló/ 'enfermo’ [na.swa.ló]

vocoide muy alta, posterior, cerrada, redondeada, asilábica.

Precede a una vocal tónica.

[un] /baláw/ 'viento' [ba.láun]

vocoide cerrada, posterior, asilábica. Sigue a una vocal tónica.

\subsection{Observaciones respecto de la pronunciación}

a. En el postmargen silábico se neutralizan las oclusivas sordas con las oclusivas sonoras y la semiconsonante /w/ con la consonante $/ \mathrm{v} /$.

b. En determinadas palabras se produce alternancia de fonemas. Por ejemplo:

\begin{tabular}{|c|c|c|c|}
\hline 1. & $/ \mathrm{m} / / / \mathrm{b} /$ & /binonó/ /minonó/ & 'pequeño' \\
\hline 2. & $/ \mathrm{v} / \sim / \mathrm{b} /$ & $/ \mathrm{va} / \mathrm{ba} /$ & 'mano' \\
\hline 3. & $/ \mathrm{k} / \sim / \mathrm{g} /$ & /kačín/ /gačín/ & ‘cuánto’ \\
\hline 4. & $/ \mathrm{ph} / \sim / \mathrm{p} /$ & /phej/ /pej/ & 'hermana' \\
\hline 8. & $/ \mathrm{th} / / \mathrm{t} /$ & $/ \operatorname{than} / \sim \tan /$ & 'cama' \\
\hline 9. & $/ \mathrm{kh} / \sim / \mathrm{k} /$ & /kham/ /kam/ & 'sol' \\
\hline 10. & /čh/ /čl & /čhučhó/ /čučó/ & ‘vacío' \\
\hline 11. & $/ \mathrm{kh} / \sim / \mathrm{x} /$ & /dikhé/ /dixé/ & '(tú) ves' \\
\hline 12. & /j/ /džl & /jek/ /džek/ & 'uno' \\
\hline
\end{tabular}

c. Las consonantes dentales $/ \mathrm{d} / \mathrm{y} / \mathrm{t} / \mathrm{se}$ pierden cuando ocurren en la misma sílaba después de otra consonante (ejemplo, /dand/ 'diente' y /baxt/ suerte, se pronuncian [dan] y [bax] respectivamente).

d. En algunos informantes se advierte una pérdida del contraste entre las consonantes puras y aspiradas.

\subsection{Respecto de la interpretación fonémica de los fonos [j] [ij] [u] y [w]}

Las siguientes razones justifican la interpretación fonémica de los fonos ([i] [i]); ([w] ([u $]$ ) como alófonos de las semiconsonantes $/ \mathrm{j} / \mathrm{y} / \mathrm{w} /$ :

a. La distribución de [j], [i], [w], [u] es típicamente consonántica: no ocupan la cima silábica y ocurren en contextos donde sólo ocurren los fonos contoides (en el pre y postmargen silábicos). 
b. En el /rromané/ de Chile, dos vocales contiguas -excepto si una de ellas es [j] [i] [w] o [u] - pertenecen siempre a sílabas distintas (/čeraí/= [če.ra.í] 'estrella'; /eá/ = [e.á] 'sí'; /ladžáo/= [la.džá.o] 'vergüenza').

c. Estos vocoides alternan libremente con contoides en determinados contextos.

\section{Fonotaxis}

\subsection{La sílaba}

Una palabra puede constar de:

Una sílaba:

Dos sílabas:

/des/ 'diez'

Tres sílabas:

/čís.me/ 'botas'

Cuatro sílabas:

lís.ti.na/ 'verdad'

/bin.di.vó.ri/ 'abeja'

El foco silábico siempre lo constituye una vocal.

Una vocal puede estar precedida en la misma sílaba por:

Una consonante:

Una semiconsonante:

Dos consonantes:

/er/ 'casa'

Una consonante y una semiconsonante:

/jag/ 'fuego'

/tró.ni/ 'resfrío'

/thjá.ra/ 'mañana'

Las posibilidades silábicas del /rromané/ de Chile son las siguientes:

1. V

2. $\mathrm{VC}$

$\mathrm{VSc}$

3. $\mathrm{CV}$

$\mathrm{ScV}$

4. $\mathrm{CVC}$

$\mathrm{CVSc}$

$\mathrm{ScVC}$

$\mathrm{ScVSc}$

5. CVCC

6. $\mathrm{CCV}$

$\mathrm{CScV}$

7. $\mathrm{CCVC}$

$\mathrm{CScVC}$

CCVC

$\mathrm{CScVSc}$ lo/

/ís.ti.na/

/áj.di/

/ko.ról

/wa/

/čir/

/paj/

/jag/

/jiw/

/burs/

/glí.bo/

/i.njá/

/trus/

/rjat/

/ka.tráv.čo/

/bjaw/ el

(artículo masc.)

verdad

¡vamos!

pulsera

eso

hormiga

agua

fuego

nieve

año

barro

nueve

sed

noche

pepino

casamiento 


\subsection{Distribución de los fonemas}

\subsubsection{Vocales}

Todas las vocales ocurren en posición inicial y final absoluta. Su frecuencia en estos contextos es baja.
1. /akór/
'nuez'
1. /akhál
'ojos'
2. lespídaw/
'(yo) empujo'
2. /telé/
'abajo'
3. /injávardes/
'noventa'
3. /džilí/
'canción'
4. /oxtóvardes/
'ochenta'
4. /čaró/
'plato'
5. /udár/
'puerta'
5. /gurú/
'toro'

\subsubsection{Consonantes y semiconsonantes}

\subsubsection{Una consonante}

En posición inicial de palabra ocurren todas las consonantes. No todas ocurren ante las cinco vocales:

\begin{tabular}{llll}
\multicolumn{2}{c}{$/ \mathrm{p} /$} & & \multicolumn{2}{c}{ /b/ } \\
/a/ /patrín/ & 'hoja' & /a/ /bal/ & 'pelo' \\
/e/ /pétalo/ & 'herradura' & /e/ /bes/ & 'isiéntate!' \\
/i/ /pisóm/ & 'pulga' & /i//bíbi/ & 'tía' \\
/o/ /póski/ & 'bolsillo' & /o/ /bóbo/ & 'choclo' \\
/u/ /purúm/ & 'cebolla' & /u/ /but/ & 'mucho'
\end{tabular}

$\begin{array}{ll}\text { /a/ /phabáj/ } & \text { 'manzana' } \\ \text { /e/ /phenáw/ } & \text { '(yo) digo' } \\ \text { /i/ /phiráw/ } & \text { '(yo) camino' } \\ \text { /o/ } / \text { /phur/ } & \text { 'puente' }\end{array}$

\begin{tabular}{lll} 
& \multicolumn{1}{c}{$/ \mathrm{t} /$} & \\
/a/ & $/ \mathrm{ta} /$ & 'y' \\
/e/ & $/ \mathrm{te} /$ & (marca de infinitivo) \\
/i/ & /tíma/ & (nombre propio) \\
/o/ & $/$ tolári/ & 'moneda' \\
/u/ & $/$ tumén/ & 'ustedes'
\end{tabular}

$\begin{array}{ll}\text { /a/ /dad/ } & \\ \text { /e/ /del/ } & \text { 'padre' } \\ \text { /i//diló(i)/ } & \text { 'loco(a)' } \\ \text { /o//dópo/'s } & \\ \text { /u//dur/ } & \text { 'lejos' }\end{array}$




$\begin{array}{ll}\text { /a/ /thaw/ } & \\ \text { /e/ /them/ } & \text { 'pilo' } \\ \text { /i// } & \\ \text { /o/ /thow/ } & \text { 'ipon!' } \\ \text { /u/ /thuló(í)/ } & \text { 'gordo(a)' (masc.) }\end{array}$

\begin{tabular}{|c|c|}
\hline \multicolumn{2}{|c|}{$/ \mathrm{k} /$} \\
\hline /a/ /karrnó/ & 'espina' \\
\hline /e/ /ke/ & 'que' \\
\hline /i/ /kisí/ & (ver nota 2 \\
\hline /o/ /kókalo/ & 'hueso' \\
\hline /u/ /kukúj/ & 'codo' \\
\hline
\end{tabular}

\begin{tabular}{|c|c|}
\hline \multicolumn{2}{|c|}{$/ \mathrm{kh} /$} \\
\hline $\begin{array}{l}\text { /a/ /kham/ } \\
\text { /e/ }\end{array}$ & 'sol' \\
\hline /o/ /khoj/ & 'grasa' \\
\hline /u/ /khul/ & 'excremento' \\
\hline
\end{tabular}

/f/

$\begin{array}{lll}\text { /a/ } & \text { /fála/ } & \text { 'gracias' } \\ \text { le/ } & - & \\ \text { /i/ } & - & \\ \text { /o/ } & \text { /fóro/ } & \text { 'ciudad' } \\ \text { /u/ } & \text { /fusúj/ } & \text { 'poroto' }\end{array}$

$/ \mathrm{s} /$

$\begin{array}{ll}\text { /a/ /sapúj/ } & \text { 'jabón' } \\ \text { /e//serán/ } & \text { 'almohada' } \\ \text { /i/ /síva/ } & \text { 'trenza' } \\ \text { /o/ /soró/ } & \text { 'cabeza' } \\ \text { /u//sunakáj/ } & \text { 'oro' }\end{array}$

$\begin{array}{ll} & \text { /g/ } \\ \text { /a/ /gadžól } & \text { 'hombre no gitano' } \\ \text { /e//geldála/ } & \text { 'espejo' } \\ \text { /i/ /gičál/ } & \text { 'hipo' } \\ \text { /o/ /gotíta/ } & \text { 'poco' } \\ \text { /u/ /guru(m)ní/ } & \text { 'vaca' }\end{array}$

/v/

la/ /va/ 'mano'

le/ /veselipé/ 'alegría'

/i/ /vizáj/ 'inteligente'

/o/ /vow/ 'él'

/u/

$|z|$

/a/ /záño/ ${ }^{6}$

/e//zeá/ 'espalda'

/i/ /zído/ 'muralla'

/o/ /zórka/

/u/ /zumí/

(nombre propio)

'sopa'

\begin{tabular}{|c|c|}
\hline \multicolumn{2}{|c|}{$|x|$} \\
\hline $\begin{array}{l}\text { /a/ /xárkuma/ } \\
\text { /e/ }\end{array}$ & 'cobre' \\
\hline /i/ /xiw/ & 'hoyo' \\
\hline $\begin{array}{l}\text { /o/ /xoljáriko/ } \\
\text { /u/ /xurguzó/ }\end{array}$ & $\begin{array}{l}\text { 'enojado' } \\
\text { 'melón' }\end{array}$ \\
\hline
\end{tabular}


|čl

$\begin{array}{ll}\text { /a/ /čára/ } & \text { 'carpa' } \\ \text { /e/ /čreráw/ } & \text { '(yo) hago' } \\ \text { /i//čirés/ } & \text { 'cereza' } \\ \text { /o/ /čokáno/ } & \text { 'martillo' } \\ \text { /u/ /čučíl/ } & \text { 'seno' }\end{array}$

/dž/

$\begin{array}{lll}\text { /a/ } & \text { /džandári/ } & \text { 'policía' } \\ \text { /e/ } & \text { /džené/ } & \text { 'personas' } \\ \text { /i/ } & \text { /džanáw/ } & \text { '(yo) sé' } \\ \text { /o/ } & \\ \text { /u/ } & \text { /džuvlí/ } & \text { 'mujer no gitana' }\end{array}$

/čh/

$\begin{array}{lll}\text { /a/ } & \text { /čhamá/ } & \text { 'mejillas' } \\ \text { le/ } & \text { /čhej/ } & \text { 'muchacha gitana' } \\ \text { /i/ } & & \\ \text { /o/ } & \text { /čhon/ } & \text { 'luna' } \\ \text { /u/ } & \text { /čhučhó/ } & \text { 'vacío' }\end{array}$

\begin{tabular}{lll}
\multicolumn{3}{c}{$/ \mathrm{m} /$} \\
/a/ & /mató(í)/ & 'borracho(a)' \\
/e/ & /meráw/ & '(yo) muero' \\
/i/ & /minía/ & 'zapato' \\
/o/ & $/$ mol/ & 'vino' \\
/u/ & /mudaráw/ & '(yo) mato'
\end{tabular}

\begin{tabular}{|c|c|c|}
\hline \multicolumn{3}{|c|}{$/ \mathrm{n} /$} \\
\hline /a/ & /naj/ & 'dedo' \\
\hline le/ & /nevó(1)/ & 'nuevo(a)' \\
\hline /i/ & /níso/ & 'nada' \\
\hline & lúko(a)/ & 'nieto(a)' \\
\hline
\end{tabular}

\begin{tabular}{|c|c|c|}
\hline \multirow{2}{*}{\multicolumn{2}{|c|}{ la/ / /ñaño/ }} & $/ \tilde{\mathrm{n}} /$ \\
\hline & & (nombre propio) \\
\hline /i/ & & \\
\hline & 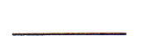 & \\
\hline \multicolumn{3}{|c|}{ /1/ } \\
\hline /a/ & /lacóóí)/ & 'bueno(a)' \\
\hline le/ & /leási/ & 'gitano argentino' \\
\hline /i/ & /limórja/ & 'cementerio' \\
\hline $10 /$ & /loló(î́)/ & 'rojo(a)’ \\
\hline$/ \mathrm{u} /$ & /luviníča/ & 'sandía' \\
\hline
\end{tabular}




\begin{tabular}{|c|c|c|}
\hline \multicolumn{3}{|c|}{$/ \mathrm{r} /$} \\
\hline$/ \mathrm{a} /$ & /rat/ & 'sangre' \\
\hline le/ & /rézo/ & 'arroz' \\
\hline /i/ & /rik/ & 'orilla, lado' \\
\hline $10 /$ & /ródaw/ & '(yo) busco' \\
\hline$/ \mathrm{u} /$ & /rup/ & 'plata' \\
\hline \multicolumn{3}{|c|}{$/ \mathrm{rr} /$} \\
\hline$/ \mathrm{a} /$ & /rra/ & 'pedo' \\
\hline /i/ & & \\
\hline /o/ & /rroj/ & 'cuchara' \\
\hline
\end{tabular}

En contexto intervocálico ocurren todas las consonantes, excepto la aspirada /ph/. En este contexto, toda consonante forma sílaba con la vocal que le sigue:

$\begin{array}{lll}\text { /p/ } & \text { /xa.pé/ } & \text { 'comida' } \\ * \mathrm{VphV} & \text { /gu.lú.bo/ } & \\ \text { /b/ } & \text { /ka.té/ } & \text { 'paloma' } \\ \text { /t/ } & \text { /gá.do/ } & \text { 'aquí' } \\ \text { /d/ } & \text { /mo.tho.dém/ } & \text { 'asco, hediondez, feo' } \\ \text { /th/ } & \text { /bu.kó/ } & \text { '(yo) hablé, dije, conté' } \\ \text { /k/ } & \text { /či.gá.ra/ } & \text { 'hígado' } \\ \text { /g/ } & \text { /li.khá/ } & \text { 'cigarro' } \\ \text { /kh/ } & \text { /ka.fá.va/ } & \text { 'liendres' } \\ \text { /f/ } & \text { /mi.rí.so/ } & \text { 'café' } \\ \text { /s/ } & \text { /ré.zo/ } & \text { 'colonia' } \\ \text { /z/ } & \text { /ko.xa.dó/ } & \text { 'arroz' } \\ \text { /x/ } & \text { /pa.ra.mí.či/ } & \text { 'mentiroso' } \\ \text { /č/ } & \text { /bu.džó/ } & \text { 'cuento' } \\ \text { /dž/ } & \text { /čhu. čhó/ } & \text { 'bolso' } \\ \text { /čh/ } & \text { /pa.mú.ko/ } & \text { 'vacío' } \\ \text { /m/ } & \text { /zi.tí.no/ } & \text { 'algodón' } \\ \text { /n/ } & \text { /bo.rá.ña/ } & \text { 'aceite' } \\ \text { /ñ / } & \text { /me.la.ló(í)/ } & \text { 'poroto verde' } \\ \text { /l/ } & \text { /bu.ré.ko/ } & \text { 'sucio(a)' } \\ \text { /r/ } & \text { /čo.rrí/ } & \text { 'ombligo' } \\ \text { /rr/ } & & \text { 'hija' } \\ & & \end{array}$

En posición final de palabra ocurren las siguientes consonantes:

Oclusivas puras

$/ \mathrm{p} /$

/b/

/sap/

/drab/

'serpiente'

'remedio' 


$\begin{array}{lll}/ \mathrm{t} / & / \mathrm{kat} / & \text { 'tijeras' } \\ / \mathrm{d} / & / \mathrm{gad} / & \text { 'camisa' } \\ / \mathrm{k} / & / \mathrm{mak} / & \text { 'mosca' } \\ / \mathrm{g} / & / \mathrm{jag} / & \text { 'fuego' }\end{array}$

Oclusiva aspirada $/ \mathrm{kh} /$

/kh/ Ilikh/ 'liendre'

Fricativa /s/

/s/ Imas/ 'carne'

En interior de palabra -en el postmargen silábico- ocurren /f/ y $/ \mathrm{x} /$ :

/f/ léf.ti.no/ 'barato'

/x/ /tax.táj/ 'vaso'

Africada /゙̌/

/č/ /kuč/ 'caro'

Nasales

$/ \mathrm{m} / \quad / \mathrm{sem} / \quad$ 'soy, estoy'

Lateral

/1/ /del/ 'Dios'

Vibrantes
$/ \mathrm{r} /$
lan.gár/
'carbón'
/rr/
/barr/
'piedra'

\subsubsection{Una semiconsonante}

En posición inicial de palabra ocurren las dos semiconsonantes, pero ninguna ocurre ante todas las vocales:

$\begin{array}{lll} & \text { /j/ } & \\ \text { /a/ } & \text { /jag/ } & \text { 'fuego' } \\ \text { le/ } & \text { /jek/ } & \text { 'uno' }\end{array}$




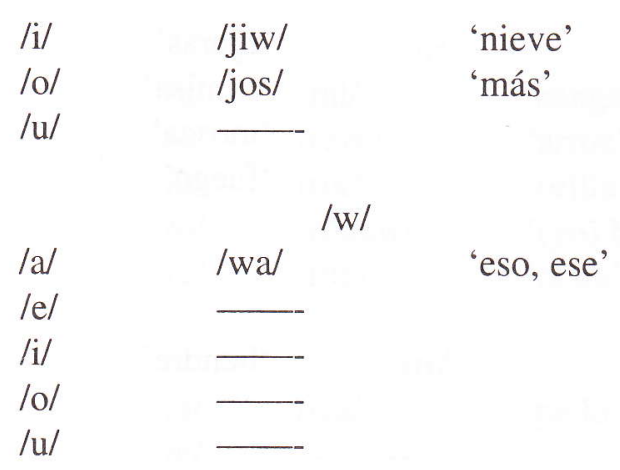

En contexto intervocálico ocurren las dos semiconsonantes. En este contexto, forman sílaba con la vocal siguiente.

$\begin{array}{lll}/ \mathrm{j} / & \text { /go.já/ } & \text { 'ésta' } \\ / \mathrm{w} / & / k a ́ . w i . c ̌ i / & \text { "una de las "razas" gitanas' }\end{array}$

En posición final de palabra ocurren:

/kaj/ 'dónde'
/sow/ 'seis'

4.2.2.3. Una consonante y una semiconsonante

En posición inicial de palabra ocurren los siguientes grupos que forman sílaba con la vocal siguiente:

\begin{tabular}{|c|c|c|c|}
\hline \multicolumn{4}{|c|}{$/ \mathrm{p}, \mathrm{b}, \mathrm{d}, \mathrm{th}, \mathrm{g}, \mathrm{l}, \mathrm{r} /+\mathrm{j} / / \mathrm{pj} /$} \\
\hline & /bj/ & /bjaw/ & 'casamiento' \\
\hline & /dj/ & /dja/ & "(él-ella) dio" \\
\hline & /thj/ & /thjára/ & 'mañana' \\
\hline & $/ \mathrm{gj} /$ & /gja/ & ‘así' \\
\hline & $/ \mathrm{lj} /$ & /ljo/ & ‘corazón’ \\
\hline & $/ \mathrm{rj} /$ & /rjat/ & 'noche' \\
\hline & $/ \mathrm{sw} /$ & /swéto/ & 'familia' \\
\hline
\end{tabular}

En contexto intervocálico, las semiconsonantes forman sílaba con la consonante precedente y la vocal siguiente. Algunos de los grupos más frecuentes en este contexto son los siguientes:

$$
\begin{aligned}
& \text { /s,n,m,r, } 1 /+/ \mathrm{j} / \\
& \text { /V.sjV/ } \\
& \text { /V.njV/ } \\
& \text { /mi.sjá.ko/ } \\
& \text { 'ratón' } \\
& \text { 'vestido' }
\end{aligned}
$$




$\begin{array}{llll} & \text { /V.mjV/ } & \text { /rro.mjá/ } & \text { 'esposas (gitanas)' } \\ & \text { /V.rjV/ } & \text { /ba.rjá.ko/ } & \text { 'mástil' } \\ \text { /xo.ljá.ri.ko/ } & \text { 'enojado' } \\ \text { / /s/ }+ \text { /wV/ } & \text { / } & \text { /na.swa.ló/ } & \text { 'enfermo' }\end{array}$

En posición final de palabra no ocurre este grupo.

\subsubsection{Una semiconsonate más una consonante}

Este grupo sólo ocurre en contexto intervocálico con la semiconsonante /j/. En este caso, ésta pertenece a la sílaba de la vocal precedente y la consonante a la sílaba de la vocal siguiente (ej. /kaj.má.ko/ 'mantequilla', /de.vój.ka/ (nombre propio)).

\subsubsection{Dos consonantes}

En posición inicial de palabra ocurren los siguientes grupos:

Oclusivas puras $+/ \mathrm{r} /$

\begin{tabular}{|c|c|c|c|}
\hline & $/ \mathrm{pr} /$ & /prá.xo/ & 'ceniza' \\
\hline & /br/ & /bré.ča/ & ‘saco’' \\
\hline & $/ \mathrm{tr} /$ & $/$ trin/ & 'tres' \\
\hline & $/ \mathrm{dr} /$ & /drak/ & 'uva' \\
\hline & $/ \mathrm{kr} /$ & /kris.té.ñe/ & 'bautizo' \\
\hline & /gr/ & /gras.ní/ & ‘yegua’ \\
\hline \multicolumn{4}{|l|}{$/ \mathrm{ph} /+/ \mathrm{r} /$} \\
\hline \multirow{2}{*}{$|\mathrm{f} /+| \mathrm{r} /$} & $/ \mathrm{phr} /$ & /phral/ & 'hermano' \\
\hline & $/ \mathrm{fr} /$ & /frín.ka/ & (nombre propio) \\
\hline
\end{tabular}

Oclusivas puras bilabiales y velares $+/ 1 /$

\begin{tabular}{|c|c|c|c|}
\hline & $/ \mathrm{pl} /$ & /pla.ti.já.ja/ & 'tomate' \\
\hline & $/ \mathrm{bl} /$ & /blá.ko/ & 'nube' \\
\hline & /k1/ & /klú.čo/ & 'llave' \\
\hline & /g1/ & /glí.bo/ & ‘barro’ \\
\hline & $/ \mathrm{st} /$ & /stó.ti.na/ & 'cien’ \\
\hline & $/ \mathrm{sl} /$ & /slá.va/ & 'celebración a la virgen' \\
\hline
\end{tabular}

En contexto intervocálico, la primera consonante pertenece a la sílaba de la vocal precedente y la segunda a la sílaba de la vocal siguiente. Se exceptúan de esta regla los dos últimos grupos de la serie que se presenta en seguida, en los cuales la primera y segunda consonantes pertenecen a la sílaba de la vocal siguiente. Los grupos consonánticos más frecuentes en este contexto son los siguientes: 


$$
\begin{gathered}
/ \mathrm{n} /+/ \mathrm{t}, \mathrm{d}, \mathrm{k}, \mathrm{g}, \mathrm{c}, \mathrm{d} z ̌ / \\
/ \mathrm{nt} / \\
/ \mathrm{nd} / \\
/ \mathrm{nk} / \\
/ \mathrm{ng} / \\
/ \mathrm{nc} / \\
/ \mathrm{ndž/} /
\end{gathered}
$$

$/ \mathrm{r} /+/ \mathrm{p}, \mathrm{b}, \mathrm{d}, \mathrm{k}, \mathrm{g}, \mathrm{s}, \mathrm{z}, \mathrm{v}, \mathrm{c}, \mathrm{l}$

$\begin{array}{ll}\text { /rp/ } & \text { /kar.pé.li/ } \\ \text { /rb/ } & \text { /búr.be/ } \\ \text { /rd/ } & \text { /búr.do/ } \\ \text { /rk/ } & \text { /kur.kó/ } \\ \text { /rg/ } & \text { /xur.gu.zó/ } \\ \text { /rs/ } & \text { /bur.sínd/ } \\ \text { /rz/ } & \text { /mur.zíw/ } \\ \text { /rv/ } & \text { /kur.vá.no/ } \\ & \\ \text { /rč/ } & \text { /ma.gár.čo/ } \\ \text { /rl/ } & \text { /es.ter.lí.ča/ }\end{array}$

$/ \mathrm{s} /+/ \mathrm{t}, \mathrm{d}, \mathrm{k}, \mathrm{g}, \mathrm{m}, \mathrm{n}, \mathrm{l}$

/st/

$/ \mathrm{sd} /$

/sk/

/sg/

$/ \mathrm{sm} /$

/sn/

/sl/

$/ \mathrm{l} / \mathrm{t}, \mathrm{p}, \mathrm{t}, \mathrm{d}, \mathrm{s}, \mathrm{v}, \mathrm{c}, \mathrm{m} /$

/p/
$/ \mathrm{l} /$
$/ \mathrm{ld} /$
$/ \mathrm{ls} /$
$/ \mathrm{lv} /$
$/ \mathrm{lc} /$
$/ \mathrm{lm} /$

$/ \mathrm{m} /+/ \mathrm{p}, \mathrm{b} /$ /pán.tič/

/pín.da/

/čo.ván.ka/

/an.gu.rus.tí/

/in.čá/

/lu.lun.džín/ /as.tú.ko/

/gás.da/

/dis.kí.nja/

/es.gó.de/

/dus.má.no/

/bas.nó/

/pus.lú.ko/

/pál.pa.le/

/ál.ta/

/ba.sal.dó/

/púl.so/

/gál.ve.no/

/kál.če/

/dól.ma/ (apellido gitano)

'cincuenta'

(nombre propio)

'anillo'

'allá'

'flor' 'araña'

'cejas'

'cerro'

'domingo'

'melón'

'lluvia'

'(yo) envidio/odio'

(ceremonia

tradicional)

'burro'

'trueno' 'plumón'

'dueño'

'mujer'

'cosa'

'enemigo'

'gallo'

'chaleco'

'atrás'

'ven!'

'radio'

'poste de la carpa'

'amarillo'

'pantalones'

(comida gitana)

/kum.pí.ri/

/sam.bú.ka/

'papa'

'sapo' 
$/ \mathrm{v} /+/ \mathrm{t}, \check{\mathrm{c}}, \mathrm{s} /$

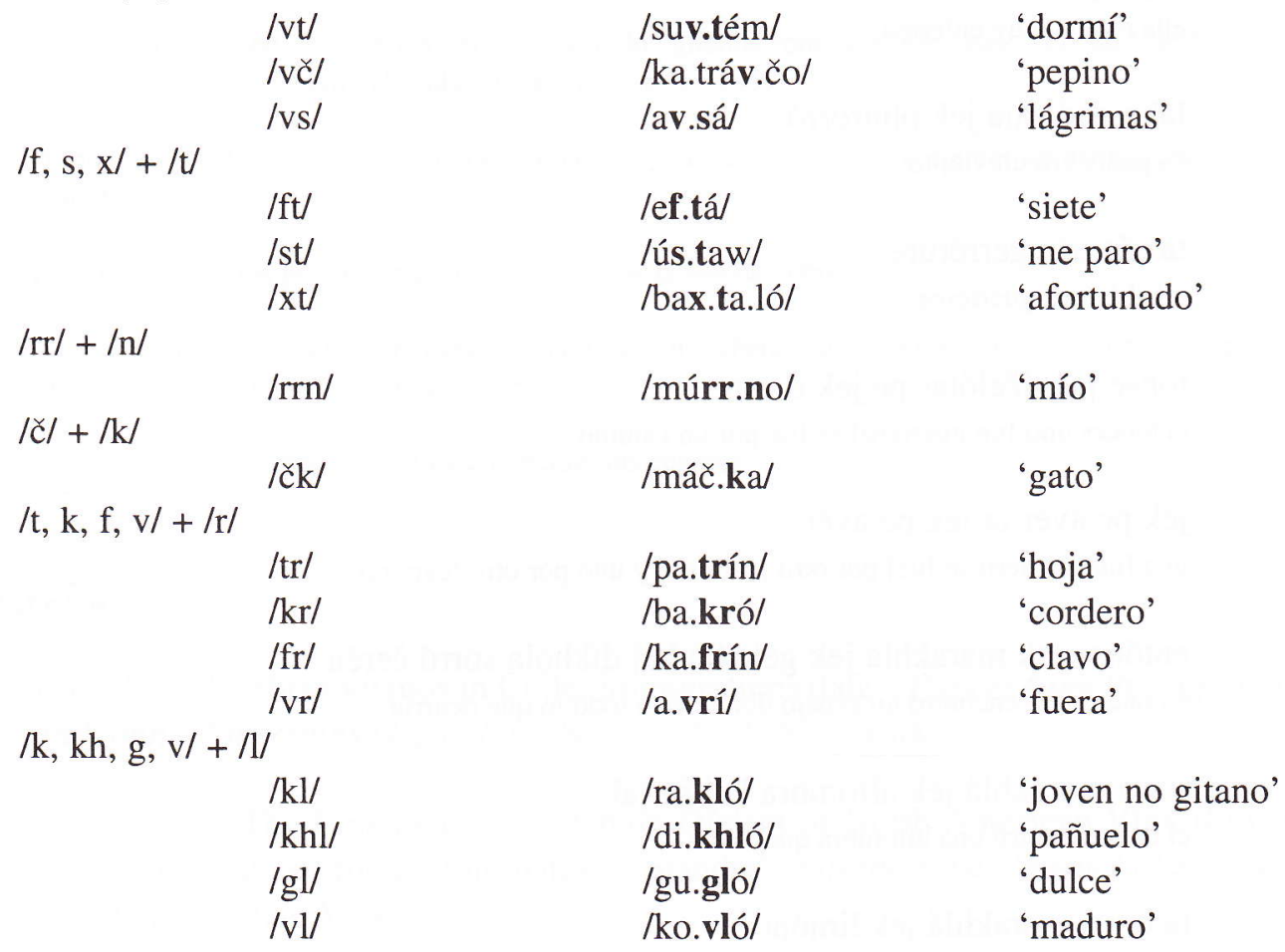

En posición final de palabra

$/ \mathrm{n} /+/ \mathrm{d}, \mathrm{g}, \check{\mathrm{c}}, \mathrm{d} \mathrm{z} /$

$\begin{array}{llll}\text { /nd/ } & \text { /dand/ } & \text { 'diente' } \\ & \text { /čang/ } & \text { 'pierna' } \\ \text { /nčl } & \text { /panč/ } & \text { 'cinco' } \\ \text { /ndž/ } & \text { /mindž/ } & \text { 'vagina' } \\ \text { /r/ /s/ } & \text { /rs/ } & \text { /burs/ } & \text { 'año' }\end{array}$

\subsubsection{Tres consonantes}

Este grupo ocurre sólo en contexto intervocálico. En esta posición, la primera consonante pertenece a la sílaba de la vocal precedente; las otras dos, a la sílaba de la vocal siguiente (/os.tro.mé/ 'afilado'; /an.dré/ 'dentro'; /am.bról/ 'pera'). Se exceptúa de esta regla la palabra /kanglí/ 'peineta', la cual se segmenta silábicamente /kang.lí/.

\section{Texto en transcripción fonémica}

sája jek rakhlí bjen sukár

Había una niña muy linda 
voj sája but naswalí

ella estaba muy enferma

láko dad sája jek phurorró

su padre era un viejito

ta séa trin gerrérure

y había tres guerreros

tonse jek dželótar pe jek drom

entonces uno [un guerrero] se fue por un camino

jek pe avér ta jek pe avér

uno [un guerrero se fue] por otro [camino] y uno por otro [camino]

entónse jek marakhlá jek geldála kaj díkhola sorró čerén

entonces uno encontró un espejo donde veía todo lo que ocurría

o wa marakhlá jek alfombra kaj fúrjal

el otro encontró una alfombra que vuela

ta o wa marakhlá jek limóno

y el otro encontró un limón

entonces pe geldála von dikhén kaj e rakhlí merél

entonces en el espejo ellos ven que la niña se está muriendo

tonse linkistén pe alfombra ta avilé čeré

entonces se subieron a la alfombra y llegaron a la casa [donde estaba la niña enferma]

koté, jek djéla o limóno ta e rakhlí džungádili

ahí, uno [un guerrero] le dio el limón y la niña se levantó

tonse e rakhlí ačilá goléa kaj djále o limóno

entonces la niña se quedó con ése que le dio el limón

porque por él se sanó

porque por él se sanó

xivíči paramíči ${ }^{7}$

\section{Notas}

1. Este grupo está establecido en Santiago de Chile. Es menos conservador en cuanto a sus costumbres y, según hemos podido establecer, habla algún dialecto rumano. 
2. Una descripción etnográfica más extensa de los gitanos se encuentra en Salamanca 1999.

3. Para una descripción etnográfica exhaustiva de los gitanos rom de Chile véase "Gitanos de Chile: Un acercamiento etnolingüístico" (Salamanca y González 1999).

4. Cartera que la gitana usa en la cintura y en la cual guarda el dinero y los elementos necesarios para "ver la suerte".

5. Herramienta que los gitanos usan para fabricar las pailas de cobre.

6. Las gitanas utilizan esta palabra para indicar a sus compañeras que deben repartir en partes iguales el dinero que obtienen cuando "ven la suerte".

7. Esta es la expresión más común para concluir sus cuentos.

\section{Bibliografía}

Dunin, Elsie. 1989. "Serbian gitanos in Chile: immigrations data". Papers from the eighth and ninth annual meetings Gypsy Lore Society. N 4. New York.

Hancock, Ian. 1993. "The Emergency of a Union Dialect of North American Vlax Romani, and Its Implications for an International Standar". International Journal of Sociology of Language. New York.

Pike, Kenneth. 1947. Phonemics. A Technique for Reducing Languages into Writing. Ann Arbor. The University of Michigan Press.

Salamanca, Gastón. 1999. “Gitanos: Algunos antecedentes etnográficos”. Sociedad Hoy. Universidad de Concepción.

Salamanca, Gastón y Alvaro González. 1999. "Gitanos de Chile: un acercamiento etnolingüístico". Atenea. Universidad de Concepción.

Vaux de F., Francoise. 1984. "De los países del indo al mundo occidental". El Correo de la Unesco. Año XXXVII: 5-7. 